\title{
28 Research Square \\ Pelvic Obliquity, It Is Not a Neutral Angle in the Normal Population
}

\author{
Alireza Moharrami \\ Tehran University of Medical Sciences \\ Seyed Saeed Tamehri \\ Tehran University of Medical Sciences \\ Mohammad Ali Ghasemi \\ Tehran University of Medical Sciences \\ Mir Mansour Moazen Jamshidi \\ Zanjan University of Medical Sciences \\ Seyed Mohammad Javad Mortazavi ( $\sim$ smjmort@yahoo.com ) \\ Tehran University of Medical Sciences
}

\section{Research Article}

Keywords: pelvic obliquity, Normal range, normal population, angle

Posted Date: July 29th, 2021

DOI: https://doi.org/10.21203/rs.3.rs-654490/v1

License: (c) (i) This work is licensed under a Creative Commons Attribution 4.0 International License. Read Full License 


\section{Abstract}

Introduction: The definition of pelvic obliquity angle (PO) still unclear in the normal population. Several factors might cause the obliquity i.e. hip osteoarthritis, developmental dysplasia of hip, trauma, scoliosis and spine related factors. Perhaps, pelvic obliquity is expressed as a deviation of neutral alignment in the normal population. We developed present study to evaluate the distribution of pelvic obliquity angle in normal population.

Method: present study retrospectively enrolled on 134 cases (70 female and 64 male) without any problem of spine, pelvic and lower extremity abnormality who referred to our institution from January 2017 to January 2018 for non-orthopedic problems. We retrospectively reviewed radiographs from institutional PACS and measured pelvic obliquity angle using mediCAD Classic software (version 3.50.0.1, Hectec, Landstuhl, Germany). Finally, all data were analyzed with SPSS software (version 24.0, USA).

Result: present study show that all data has significant relationship in one sample T test. All PO angles were normally distributed in kurtosis and skewness test. The patients were aged with a mean of 39.6 \pm 16.8 in current study. The mean of $P O$ angle was $2.18 \pm 1$.6 in all patients and 0.13 standard error of mean. There was no significant differences between male and female genders ( $2.07 \pm 1.6$ vs $2.27 \pm 1.6$, $P=0.47)$. This study reveal that age and $P O$ angle had not any correlation $(P=0.165)$.

Conclusion: despite this fact, there was an attitude that PO angle normally is in neutral alignment, our result uncover that the normal range of PO angle was not neutral and ranged from 0.58 to 4.4 degrees with one standard deviation from mean in the normal population.

\section{Introduction}

Pelvic obliquity (PO) is an unknown term in orthopedic surgery and it does not have a unique definition. One of the simple definitions for PO is a deformity that exists between the spine and pelvic axes [1] Measurement of PO is unclear just like it's definition. Fann et al discuss about the process of PO measurement by using three lines and angle of $\mathrm{PO}$ is where three lines junction [2] Zhou et al show a little different way for dimension of PO by usage of iliac crest and fourth lumbar vertebra and classified them to six groups with variation of three degrees [3]. At the same way, Torretti et al perform a study and utilize inferior part of the sacroiliac joint and the lateral edge of anterior superior iliac spine. [4]

The aim we are looking for in this study is to define a normal range of pelvic obliquity angel by measuring in people who do not have any issues that affect the PO.

\section{Material And Method}

In present study, we retrospectively enrolled 134 cases (70 female and 64 male) without any problem of spine, pelvic and lower extremity abnormality who referred to our institution from January 2017 to 
January 2018 for non-orthopedic problems.

We retrospectively extracted and reviewed pelvic radiographs from institutional PACS. All patients had standard pelvic AP (Anteroposterior) view radiograph in supine position. An expert hip and pelvic surgeon retrospectively reviewed all radiographs and confirmed that there was no abnormality and orthopaedic problem in the patients.

A standard Pelvic AP view radiography was obtained in supine position and feet rotated internally from 15 to $20^{\circ}$ (Femoral Anteversion angle correction) and greater trochanter should not overlap with femoral neck. In AP x-ray, the coccyx should be aligned with the pubic symphysis with $2.5 \mathrm{~cm}$ interval between them in the female sex and $1.5 \mathrm{~cm}$ in the male sex as described by Tannast et al [5].

Pelvic obliquity angle was measured as the angle between the horizontal plane and Hilgenreiner's line, or a line between the acetabular teardrops as described by Hagglund et al [6] using mediCAD Classic software (version 3.50.0.1, Hectec, Landstuhl, Germany). Finally, all data were analyzed with SPSS software (version 24.0, USA) and Kurtosis and skewness tests were used to evaluate normally distribution in the samples.

The Institutional Review Board (IRB) reviewed and approved the study design and confirm that there was no ethical concern in present study.

\section{Result}

We included 134 patients (70 female and 64 male) who had standard and normal AP pelvic radiograph without any orthopaedic and spine problems. The study show that all data has significant relationship in one sample T test and All PO angles were normally distributed in kurtosis and skewness test. The patients were aged with a mean of $39.6 \pm 16.8$ in current study. The mean of PO angle was $2.18 \pm 1.6$ in all patients and 0.13 standard error of mean (Fig. 1). There was no significant differences between male and female genders $(2.07 \pm 1.6$ vs $2.27 \pm 1.6, P=0.47)$. This study reveal that age and $P O$ angle had not any correlation $(P=0.165)$. (Table 1$)$

Table 1

Pelvic Obliquity Angle in Normal Population

\begin{tabular}{|lllll|}
\hline Mean & Std. Deviation & Std. Error of Mean & Minimum & Maximum \\
\hline 2.1807 & 1.606 & 0.1387 & 0 & 6.62 \\
\hline
\end{tabular}

\section{Discussion}

Different studies had a various explanation for PO, therefore, Challenge of a clear definition for PO is still remaining. The distinctive way for measurement of PO is uncertain just like the definition of it. Some studies show the procedure which we can use for prediction of PO angel, for instance, is defined as a malformation exists between the pelvis and spine axes or where inferior part of the sacroiliac joint and 
the lateral edge of anterior superior iliac spine intersect. We developed present study to evaluate the distribution of pelvic obliquity angle in normal population.

In current study, we investigate that 0.58 to 4.4 degrees defined as the normal range of PO with a mean of $2.18 \pm 1.6$ degrees and what PO degrees that not included in this range considered as an abnormal one. All discussions in different studies are about the measurement of PO and the importance of PO changes in patients with various problems that affect it. Which issue that did not give attention to and did not discuss is what the normal range of PO is or we must consider zero degrees as normal PO degrees. In spite of other literature that Zero degrees was a reference of normal PO but our study demonstrates a different result as a normal range of $\mathrm{PO}$.

In this study, PO was measured as the angle between the angle between the horizontal plane and Hilgenreiner's line, or a line between the acetabular teardrops as described by Hagglund et al [6]. There are several method to describe PO angle. A review study which did by Dubousset et al discuss about PO simply as a deformity that discovers among spine and oblique axes but the emphasis on axes that must be considered due to spine and pelvis are three-dimensional structures. He also defined PO in three ways that can explain briefly: 1 . a plastic bone and may be inaccurate with growing 2 . intercalary bone between the trunk and lower limbs 3. one unique vertebra that can be displaced in three dimensions [1]. The next study which performed by Fann et al [2] show PO angle with using three lines. Line A signifies the line drawn parallel to the line of eburnation or the intersulcate line. Lines $B$ is drawn parallel to the plumb line through the apex of each femoral head. Line $C$ is perpendicular to the plumb line. The height between line $B$ and Where Line $A$ and line $C$ meet each other is PO quantity. Zhou et al [3] revealed a simple method, as well as Torretti et al [4], discuss. Their method describes by two lines which one line linked the top of both iliac crests on AP radiography of pelvis and the second was the lowest part of the fourth lumbar vertebra and PO angel defined where two lines intersect. This method couldn't be used when lower lumbar spine is not clear and in this situation the angle between Hilgenreiner's line and horizontal lines is the best alternative method for measuring PO angel. Zhou et al also categorized the angel that calculated into 6 groups with the variation of three degrees which its start with zero degrees and defined $A$ and $B$ and their definition depends on side of PO which deviates to right or left.

Some causes of PO are contracture the muscle that relates to hip joint, length of both legs are not similar, spine deformity and problems that affect the pelvis and combination of these reasons. If we notice PO we must do the examination of hip and spine and perform radiography of both to understand the reason(s) which have different treatments such as fix the spine deformity, make a balance between both legs.

\section{Conclusion}

Despite this fact, there was an attitude that PO angle normally is in neutral alignment, our result uncover that the normal range of $\mathrm{PO}$ angle was not neutral and ranged from 0.58 to 4.4 degrees with one standard deviation (1.6 degree) from 2.18 degree as a mean in the normal population. 


\section{Declarations}

Ethics approval and consent to participate: informed written consent was obtained from the participants included in the study. The Inestitutional Review Board (IRB) of Tehran University of Medical Sciences (Imam Khomeini Complex Hospital) Reviewed and Approaved our study protocole and declared that there was no ethical Concern with the study. We Confirmed that all participants were above 16 years of age in present study.

accordance statement: all methods were carried out in accordance with relevant guidelines and regulations

Consent for publication: Written consent was obtained from our patient to publish his clinical information.

Availability of data and materials: not applicable

Competing interests: The authors declare that they have no conflict of interest.

Funding: there is no funding source

Authors' contributions: S.M.J.M supervised the study and revised the manuscript. M.G and MM.MJ performed the Xray Review. A.M reviewed the literature, prepared the manuscript. S.S.T reviewed the literature, M.M, prepared the manuscript. All authors have read and approved the manuscript

Acknowledgements: not applicable.

There are no conflict of interests to declare.

\section{References}

1. Dubousset J: Pelvic obliquity: a review. Orthopedics 1991, 14(4):479-481.

2. Fann AV, Lee R, Verbois GM: The reliability of postural x-rays in measuring pelvic obliquity. Archives of physical medicine and rehabilitation 1999, 80(4):458-461.

3. Zhou X, Wang Q, Zhang X, Chen Y, Shen H, Jiang Y: Effect of pelvic obliquity on the orientation of the acetabular component in total hip arthroplasty. The Journal of arthroplasty 2012, 27(2):299-304.

4. Torretti JA, Segal LS, Brenneman RE, Shrader MW, Chinchilli VM, Vanderhave KL: Evaluation of a Novel Method for Determining Transverse Plane Pelvic Obliquity. Spine deformity 2014, 2(1):48-54.

5. Tannast M, Siebenrock KA, Anderson SE: Femoroacetabular impingement: radiographic diagnosiswhat the radiologist should know. AJR Am J Roentgenol 2007, 188(6):1540-1552.

6. Hägglund G, Goldring M, Hermanson M, Rodby-Bousquet E: Pelvic obliquity and measurement of hip displacement in children with cerebral palsy. Acta orthopaedica 2018, 89(6):652-655. 
Figures

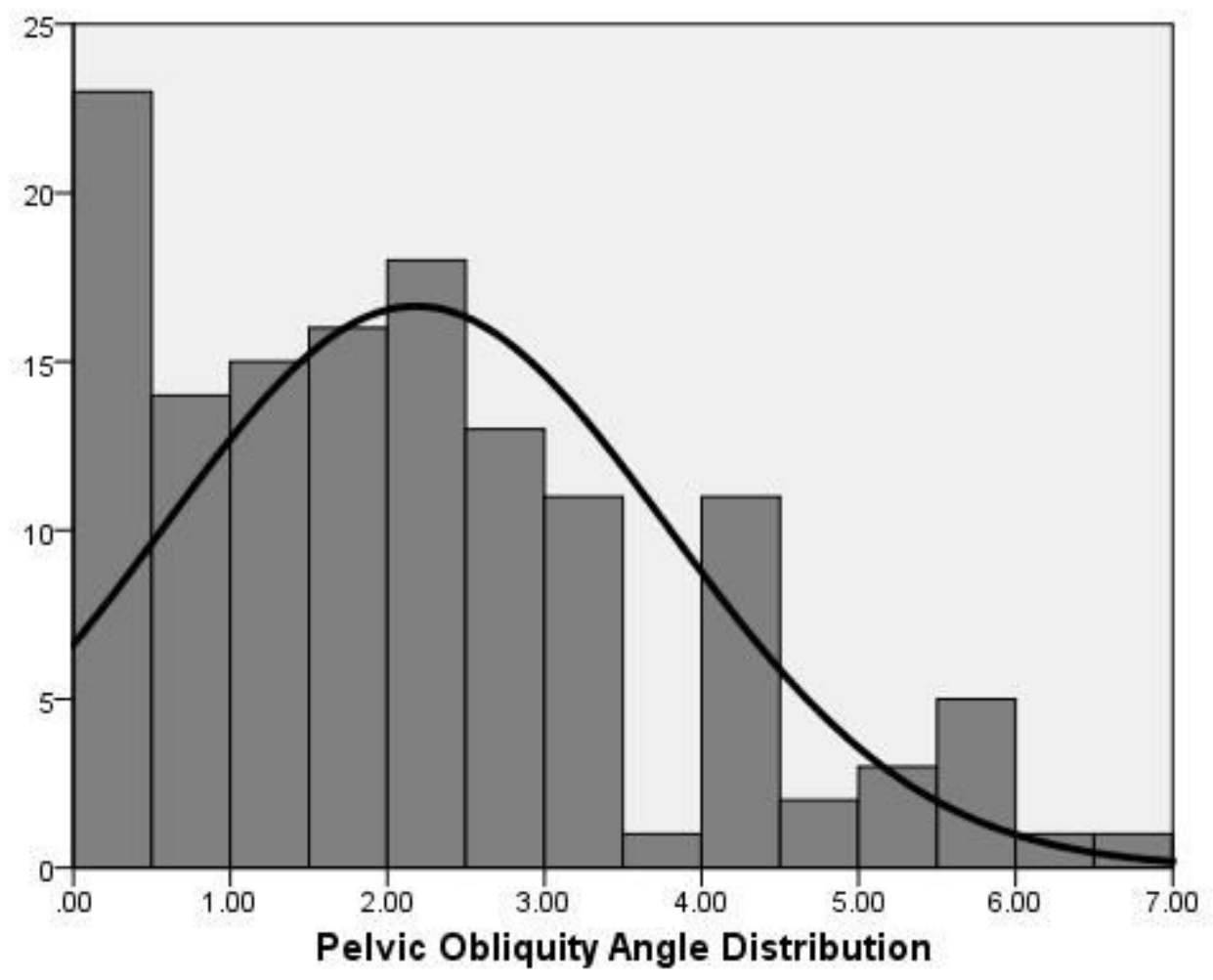

Figure 1

the Normal Distribution of Pelvic Obliquity Angle 